\title{
Effect of the Reinforce Process on the Fatigue Fracture Properties of Aeronautical Polymethyl Methacrylate
}

\author{
Wei Shang, ${ }^{1}$ Xiao-jing Yang, ${ }^{2}$ Xin-hua Ji, ${ }^{3}$ and Zhong-xian Liu ${ }^{1}$ \\ ${ }^{1}$ Key Laboratory of Protection and Retrofitting for Civil Building and Construction of Tianjin, Tianjin Chengjian University, \\ Tianjin 300384, China \\ ${ }^{2}$ School of Materials Science and Engineering, Hebei University of Technology, Tianjin 300130, China \\ ${ }^{3}$ Department of Mechanics, Tianjin University, Tianjin 300072, China
}

Correspondence should be addressed to Wei Shang; wshang@tju.edu.cn

Received 11 March 2016; Accepted 1 August 2016

Academic Editor: Pavel Lejcek

Copyright (C) 2016 Wei Shang et al. This is an open access article distributed under the Creative Commons Attribution License, which permits unrestricted use, distribution, and reproduction in any medium, provided the original work is properly cited.

\begin{abstract}
The main purpose of the present work is to investigate fatigue fracture mechanical properties of aeronautical directional polymethyl methacrylate (PMMA) which has been treated by the directional tensile technology. Firstly, the fatigue cracks growth has been tracked in directional PMMA using the stroboscopic method. Then, the digital photoelasticity with a phase-shifting technique was used to measure the uniformity of the interlaminar stretching. Based on obtained experimental results, the effect of the directional stretching on fatigue fracture properties of PMMA was analyzed. Finally, fatigue fracture properties of laminated directional PMMA were discussed and compared with those of monolayer directional PMMA. Results of this work show that the lamination technology can strengthen the interlayer of directional PMMA and enhance its fatigue fracture properties.
\end{abstract}

\section{Introduction}

As early as 1949 , Sauer and Hsiao [1, 2] began to study the crazing in the polymer. At that time, it was known that stretched areas of polymethyl methacrylate (PMMA) installed on the top of aircraft cockpit canopies had better anticraze properties. Thus, researchers from National Institute of Standards and Technology of United States embarked on the development of multiaxially and biaxially hot-stretched PMMA. In 1953, they successfully manufactured apparatus for the biaxial stretching and production of directional PMMA sheets. Since then, directional PMMA has been extensively used in the manufacture of transparent parts of aircraft canopies. The directional stretching is conducted as follows: firstly, PMMA sheets are heated to a temperature which is slightly higher than its glass transition temperature. Then, these sheets are immediately put into the stretching equipment with fixed fixtures and water-cooling devices. After being stretched to the required strain, the stretching process is over and sheets are cooled under the certain tensile force. Finally, directional PMMA sheets are obtained whose molecular chains are in an oriented state. Since directional PMMA has oriented molecular chains, the properties are significantly improved to unstretched PMMA.

Fatigue fracture properties of transparent parts of aircraft canopies play a crucial role in the safe flight performance of an aircraft. Mukherjee and Burns [3] proposed that fatigue behaviors of PMMA were determined by three parameters: stress intensity factor amplitude, average stress intensity factor, and frequency. Cheng et al. [4] studied the influence of the temperature and frequency on the fatigue crack propagation behavior of PMMA. Kim [5, 6] proposed that the fatigue crack growth rate of most polymers increased with the temperature increasing and decreased with the loading frequency increasing. Ramsteiner and Armbrust [7] solved some fatigue experimental questions of polymers such as the measurement of the crack propagation, the influence of the specimen shape and the applied frequency, and the measurement with the constant or increasing stress intensity amplitude. Yao et al. $[8,9]$ investigated the dynamic fracture behavior of thin PMMA plates with the assistance of a highspeed camera. Sahraoui et al. [10] measured the dynamic 


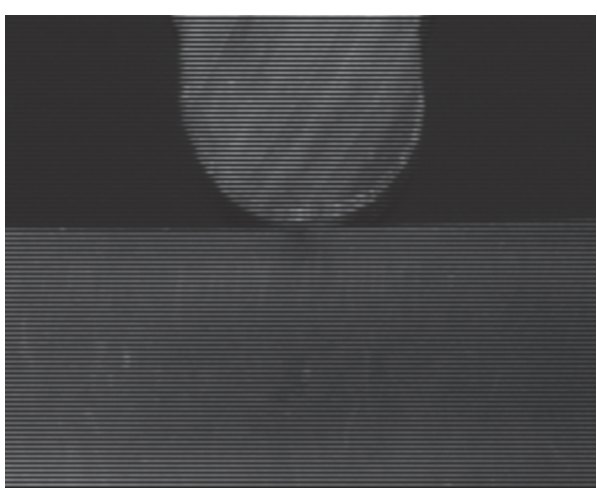

(a)

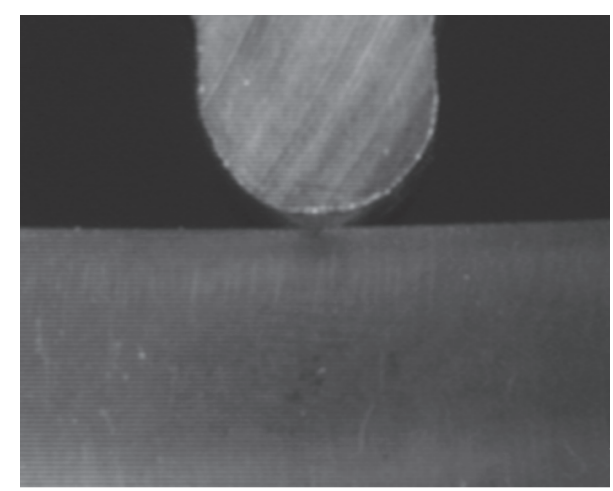

(b)

FIGURE 1: Gray distribution images of the specimen surface. (a) One acquisition; (b) twice light intensity superposition.

fracture toughness of notched PMMA at high impact velocities. The direct measurements of the specimen deflection are successfully used for the toughness evaluation. Liu et al. [11] investigated evolution of ratcheting strains of PMMA at different temperatures and stress levels by the experiment.

Currently, fatigue properties of PMMA were studied primarily based on the isotropic assumption. The author has proposed an anisotropic mechanical model for directional PMMA [12]. This research tracked the growth of fatigue cracks in directional PMMA by a stroboscopic method. Then, the digital photoelasticity with a phase-shifting technique was used to test the uniformity of the interlaminar stretching. Finally, based on obtained experimental results, the effect of the directional stretching on fatigue fracture properties of directional PMMA was analyzed.

\section{Test of Fatigue Fracture Properties by Stroboscopic Method}

The stroboscopic method is based on the following principle. The flash frequency is adjusted to be equal to the motion frequency of the tested object, so that the same position of the tested object is seen every time. For periodic motion specimens, when they are exposed to the stroboscopic light with extremely short duration pulses at a flash frequency equal to the frequency of the motion, the specimens always reach the same position every time. Therefore, the obtained picture is a stationary image (i.e., it is position invariant). When the lighting time is short enough, moving specimens can only move within a small range and can be regarded as the stationary. In this way, we can obtain images of specimens at the moment of the movement.

The stroboscopic method does not require a high-speed camera. A simple charge coupled device (CCD) camera can be used. The CCD camera generally has exposure times of approximately $30 \mathrm{~ms}$, so images of moving specimens are blurred and cannot reflect the movement of specimens at the certain position. The stroboscopic method can be used to solve this problem. According to the exposure time of the CCD camera, the stroboscopic light flashes for a very short time and can be adjusted to the duration of less than $10 \mu \mathrm{s}$.
Owing to the short illumination period, the CCD camera cannot acquire the sufficient light to produce clear images in a stroboscopic flash. Therefore, the CCD camera can acquire the sufficient light to produce clear images after many flashes at the same position. Currently, the stroboscopic method has been successfully applied to investigate the vibration of microelectromechanical systems (MEMS) [13-16].

With the increase in number of cycles, positions of the specimen change under the fatigue load; however, since the test is conducted over a long time, positions of the specimen can be considered as invariant at the same moment in adjacent cycle periods. Therefore, we can superimpose images acquired at the same moment in adjacent cycle periods. According to the above requirements, the image acquisition software was programmed based on the principle of the intensity superposition. During the illumination process, one frame image was collected, followed by another frame image. Then, gray values of these two images at corresponding positions were superimposed. This process was repeatedly conducted until clear images were acquired. Figure 1(a) shows images obtained through one acquisition, and Figure 1(b) shows twice light intensity superposition. As seen, the gray distribution of the image obtained through two superposition operations is clearer than that acquired through one.

The stroboscopic test system for fatigue fracture properties measurement is shown in Figure 2. It is comprised of a fatigue testing machine, an image acquisition system, and a synchronous control system. The image acquisition system consists of a stroboscopic light source and a CCD camera. The resolution is the number of the pixels of CCD when it is used to collect images. The resolution of using 8-bit CCD is $512 \times$ 512 pixels. Due to the application of the stroboscopic method, the normal photograph mode can meet requirements of the image acquisition.

A three-point bending fatigue test was performed on a biaxially directional PMMA specimen with a semicircular notch formed by the stress concentration in the fatigue testing machine. The length of the specimen was $100 \mathrm{~mm}$, the width was $22 \mathrm{~mm}$, the thickness was $22 \mathrm{~mm}$, and the radius of the notch was $1 \mathrm{~mm}$. The sinusoidal load with the maximum of $3 \mathrm{kN}$ and the minimum of $1 \mathrm{kN}$ was applied at a frequency 


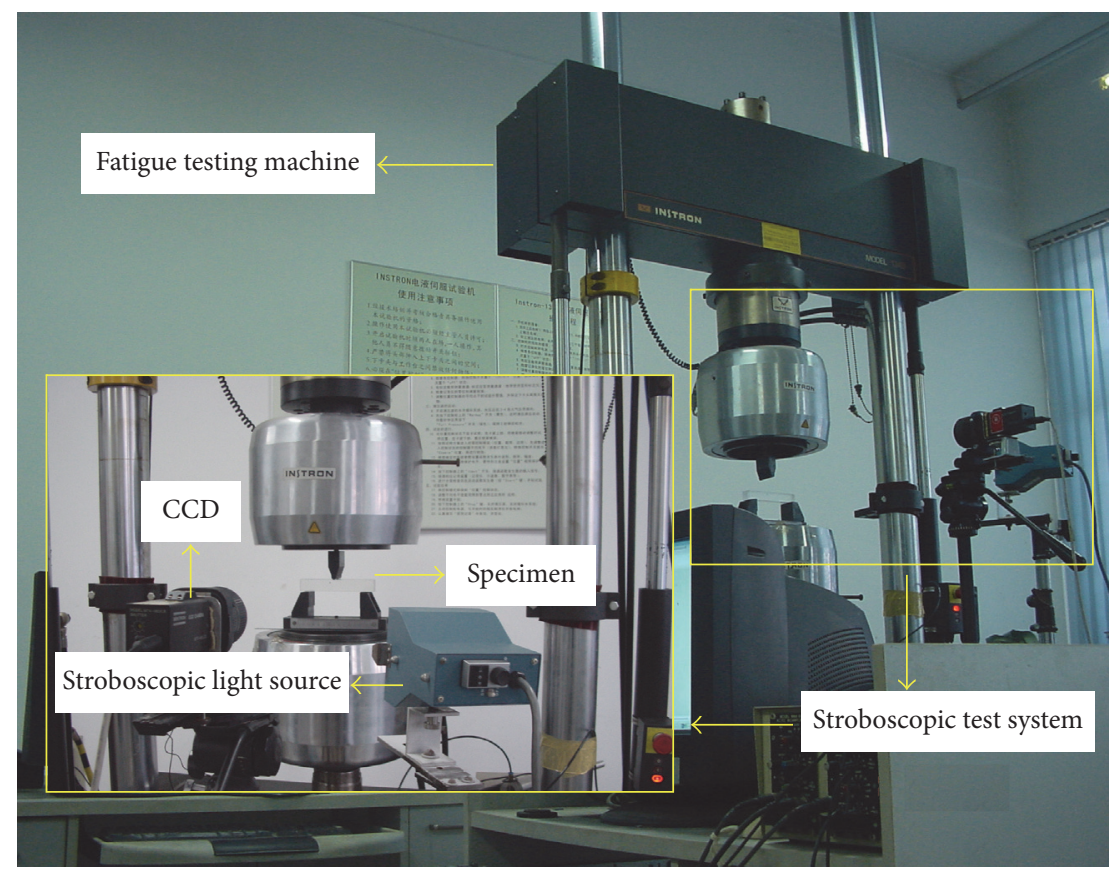

FIGURE 2: Fatigue test system for the stroboscope method.

of $1 \mathrm{~Hz}$. Meanwhile, the frequency of the stroboscopic light was adjusted to $1 \mathrm{~Hz}$. The acquisition frequency of the CCD camera was also set to $1 \mathrm{~Hz}$. They were synchronously controlled using the synchronous control system. In this way, gray distribution images of the surface of the specimen at the same region in different cycles can be acquired as shown in Figure 3. In the area with the concentrated stress, the fatigue cracks tended to grow along the direction of the tilt rather than in the vertical direction.

The fracture morphology is a record of the material fracture. The analysis of the fracture morphology is used to analyze the fracture process according to the observation and analysis of macroscopic and microscopic characteristics. The macroscopic fracture morphology of the specimen is shown in Figure 4. The fracture surface of the specimen revealed bright crystallization indicating a brittle fracture. The oval area appearing at the notch of the specimen was the area in which the crack initiation occurred. Since the crack growth can affect the transmittance of transparent materials, the stroboscopic method can be used to capture the crack growth in the transparent material.

\section{Analysis of Fatigue Crack Propagation}

The internal stress can reflect the uniformity of the tension for directional PMMA. The photoelasticity has been widely used in the measurement of the internal stress of the polymer [17]. This section investigated the phase difference of the birefringence effect in directional PMMA using the digital photoelasticity with a phase-shifting technique. The internal stress is related to the phase difference; thus the phase difference of the birefringence effect can be used to reflect the uniformity of the tension. Figure 5 shows the arrangement
TABLE 1: Optical arrangements for the eight-step phase-shifting technique.

\begin{tabular}{lcccc}
\hline Step & $\xi$ & $\eta$ & $\beta$ & Intensity equation \\
\hline 1 & $3 \pi / 4$ & $\pi / 4$ & $\pi / 2$ & $I_{1}=I_{b}+0.5 I_{a}(1+\cos \delta)$ \\
2 & $3 \pi / 4$ & $\pi / 4$ & 0 & $I_{2}=I_{b}+0.5 I_{a}(1-\cos \delta)$ \\
3 & $3 \pi / 4$ & 0 & 0 & $I_{3}=I_{b}+0.5 I_{a}(1-\sin \delta \sin 2 \theta)$ \\
4 & $3 \pi / 4$ & $\pi / 4$ & $\pi / 4$ & $I_{4}=I_{b}+0.5 I_{a}(1+\sin \delta \cos 2 \theta)$ \\
5 & $\pi / 4$ & 0 & 0 & $I_{5}=I_{b}+0.5 I_{a}(1+\sin \delta \sin 2 \theta)$ \\
6 & $\pi / 4$ & $3 \pi / 4$ & $\pi / 4$ & $I_{6}=I_{b}+0.5 I_{a}(1-\sin \delta \cos 2 \theta)$ \\
7 & $\pi / 2$ & $\pi / 4$ & 0 & $I_{7}=I_{b}+0.5 I_{a}(1-\cos \delta \sin 2 \theta)$ \\
8 & 0 & 0 & $\pi / 4$ & $I_{8}=I_{b}+0.5 I_{a}(1+\cos \delta \cos 2 \theta)$ \\
\hline
\end{tabular}

of the circular polariscope, which is the optical path of the digital photoelasticity by the eight-step phase-shifting technique. The light intensity is summarised in Table $1[18,19]$.

From intensity equations, isoclinic and isochromatic parameters are obtained as

$$
\begin{aligned}
& \theta=0.5 \tan ^{-1}\left(\frac{I_{5}-I_{3}}{I_{4}-I_{6}}\right), \text { when } I_{4} \neq I_{6}, \\
& \theta=0.5 \tan ^{-1}\left(\frac{I_{5}-I_{7}}{I_{8}-I_{6}}\right), \text { when } I_{4}=I_{6}, I_{6} \neq I_{8}, \\
& \theta=\frac{\pi}{4} \text { or } \frac{3 \pi}{4}, \quad \text { when } I_{4}=I_{6}, I_{6}=I_{8}, \\
& \delta=\tan ^{-1}\left[\frac{\left(I_{5}-I_{3}\right) \sin 2 \theta+\left(I_{6}-I_{4}\right) \cos 2 \theta}{I_{1}-I_{2}}\right],
\end{aligned}
$$




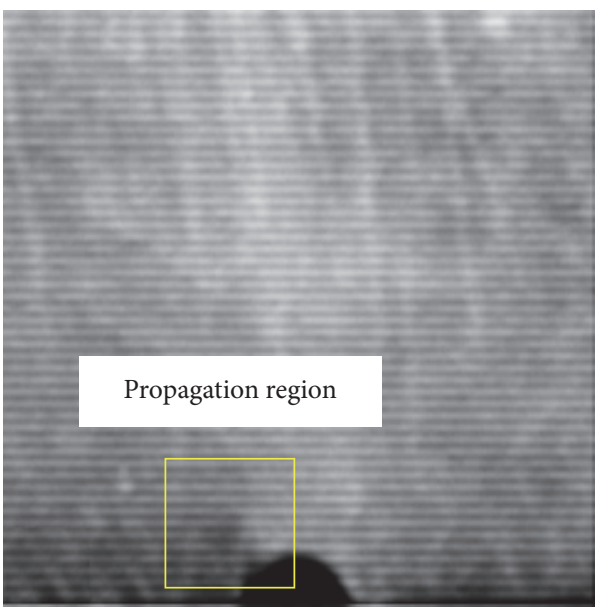

(a)

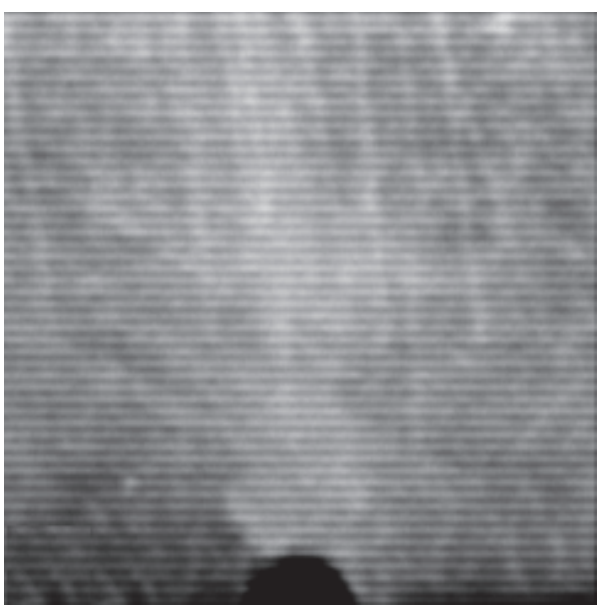

(c)

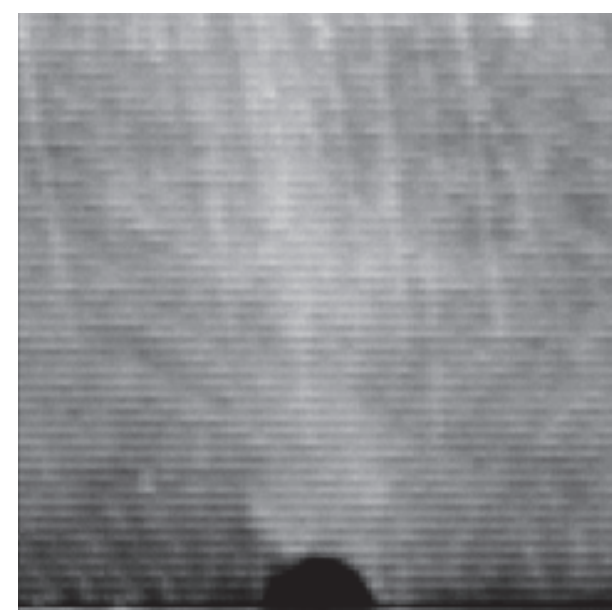

(b)

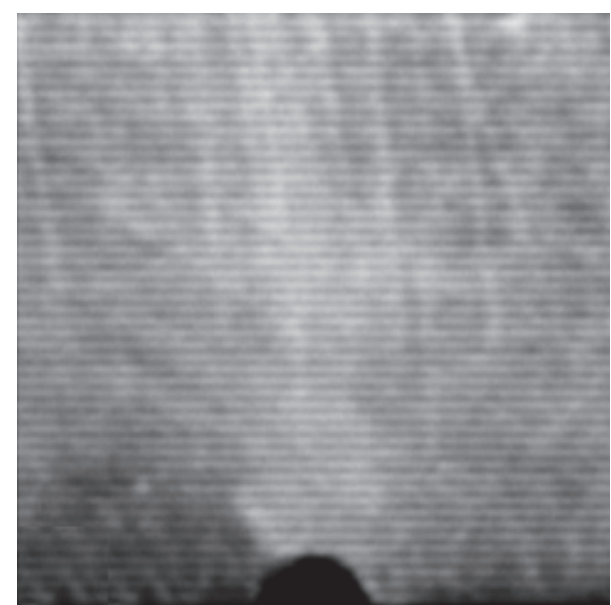

(d)

FIGURE 3: Gray distribution images at the same moment in different cycles.

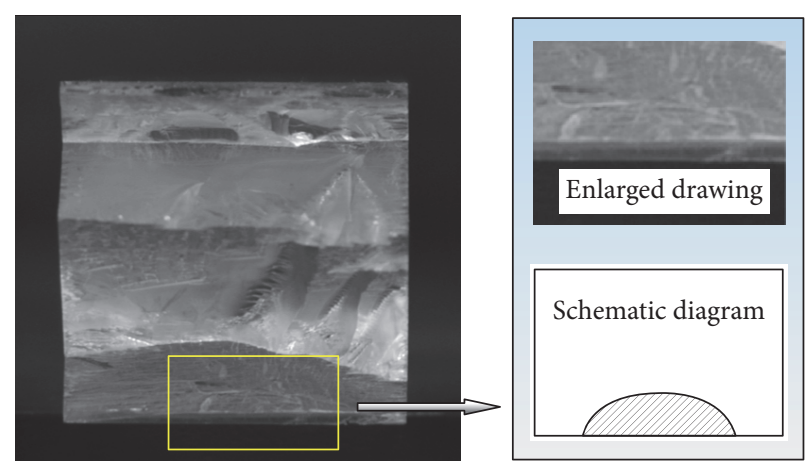

FIGURE 4: Macroscopic fracture morphology.

where $\theta$ is the isoclinic parameter, which represents the principal stress direction. $\delta$ is the isochromatic parameter, which represents the principal stress difference.

Acquired phase-shifting photoelastic images are shown in Figure 6. Based on the gray variation, the digital photoelasticity with the eight-step phase-shifting technique was used to calculate the phase difference of the birefringence effect for different layers of the sheet along the thickness direction shown in Figure 7. Results indicate that different layers of the directional PMMA sheet were stretched to different extents. The reason for this phenomenon is that when the specimen is relatively thick, it is difficult to guarantee the uniform 


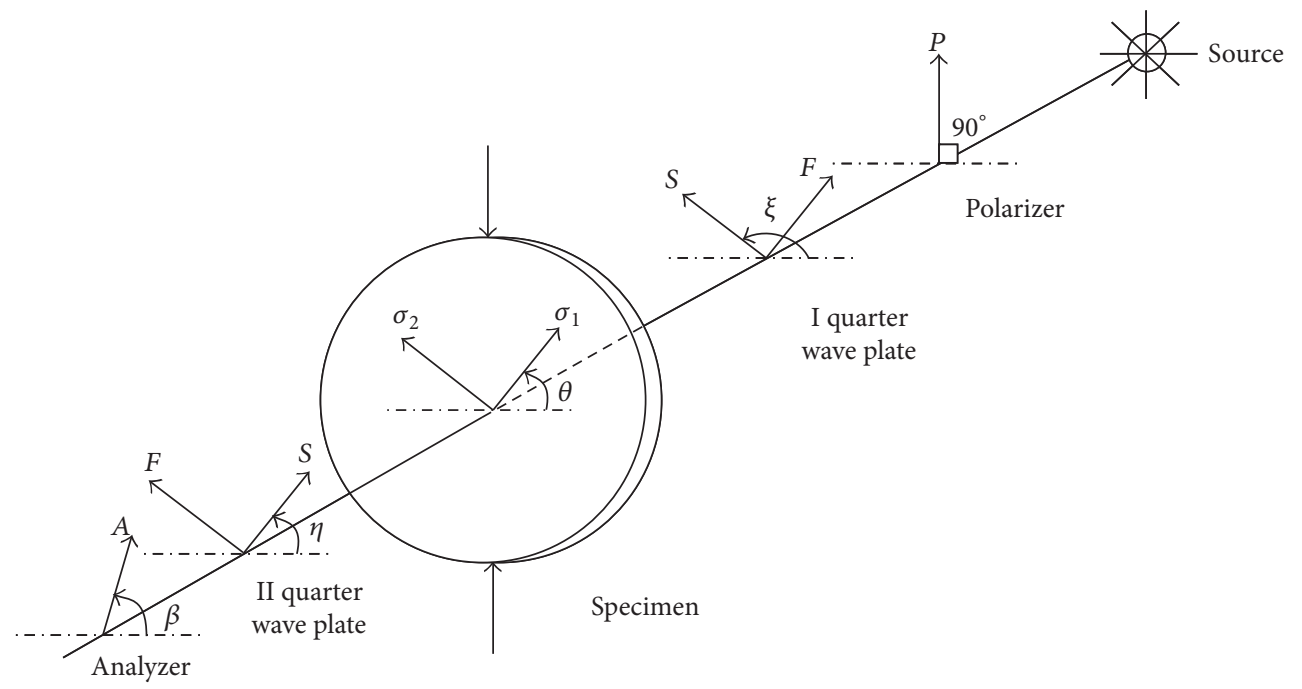

FIGURE 5: Arrangement of the circular polariscope.
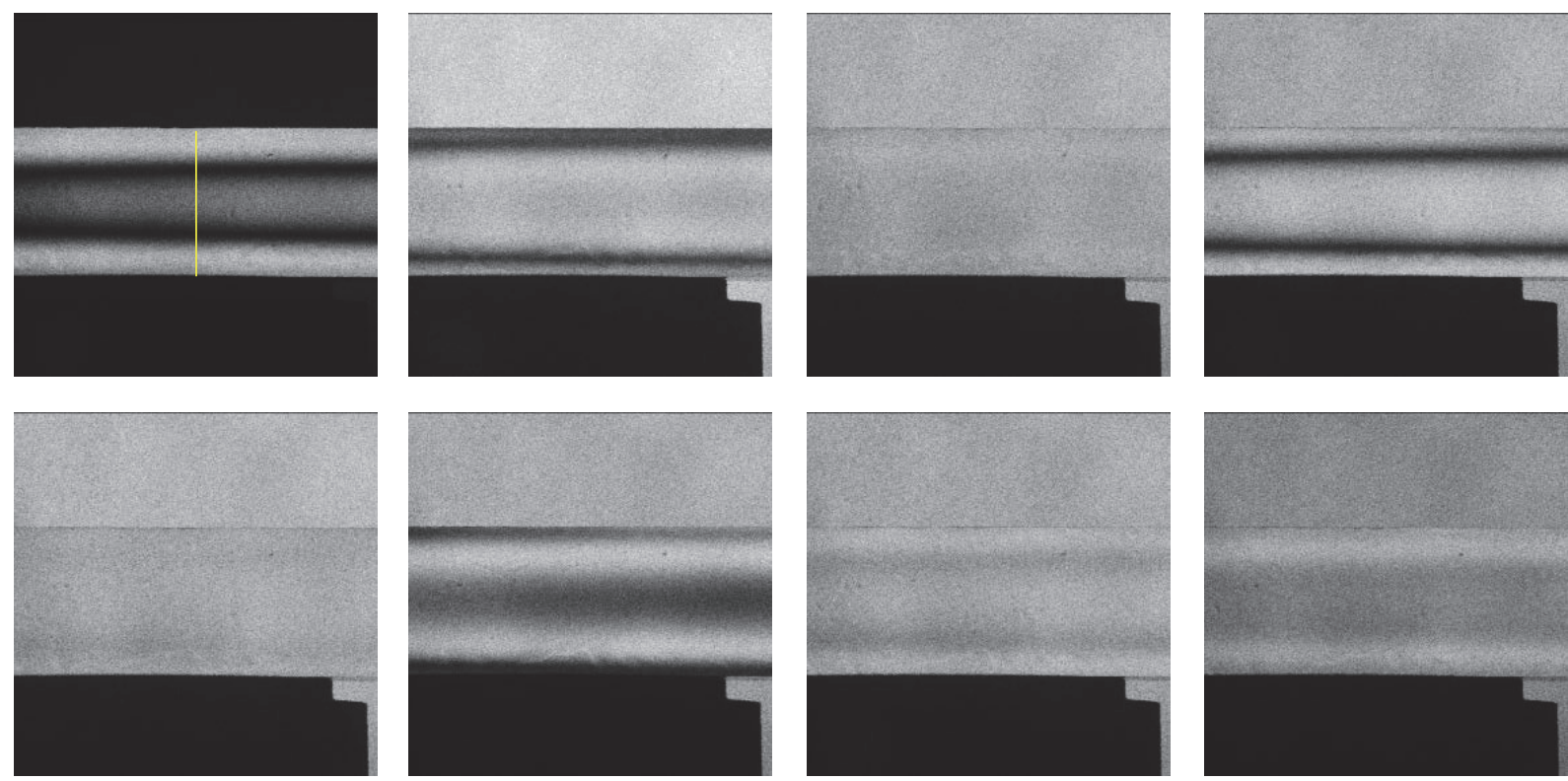

FIGURE 6: Phase-shifting photoelastic images of monolayer directional PMMA.

tension in each layer along the thickness direction. Under such circumstances, mechanical properties of each layer are inconsistent, which may cause the interlaminar cracking. As a result, the interlaminar strength is decreased. This phenomenon can explain the fatigue cracks growth along the tilting direction rather than in the vertical direction. In addition, directional PMMA was stretched at a temperature below its glass transition temperature, so molecular chains along the stretching direction became ordered and oriented. Mechanical properties of directional PMMA were improved. However, since molecular chains were not stretched along the thickness direction of the sheet, the strength was lower in that direction, which caused the growth of the fatigue crack along the tilting direction.

\section{Fatigue Fracture Properties of Laminated Directional PMMA}

Aiming at weak interlaminar mechanical properties of directional PMMA, this section used the laminate technology to improve its mechanical properties. The outer layers of laminated directional PMMA were composed of directional PMMA, while the interlayer was made of polyurethane. Laminated directional PMMA was produced by solidifying the polyurethane prepolymer and chain extender which were injected into the interlayer of directional PMMA.

The size of the laminated directional PMMA sample was the same as the monolayer directional PMMA. The length of the specimen was $100 \mathrm{~mm}$, the width was $22 \mathrm{~mm}$, 


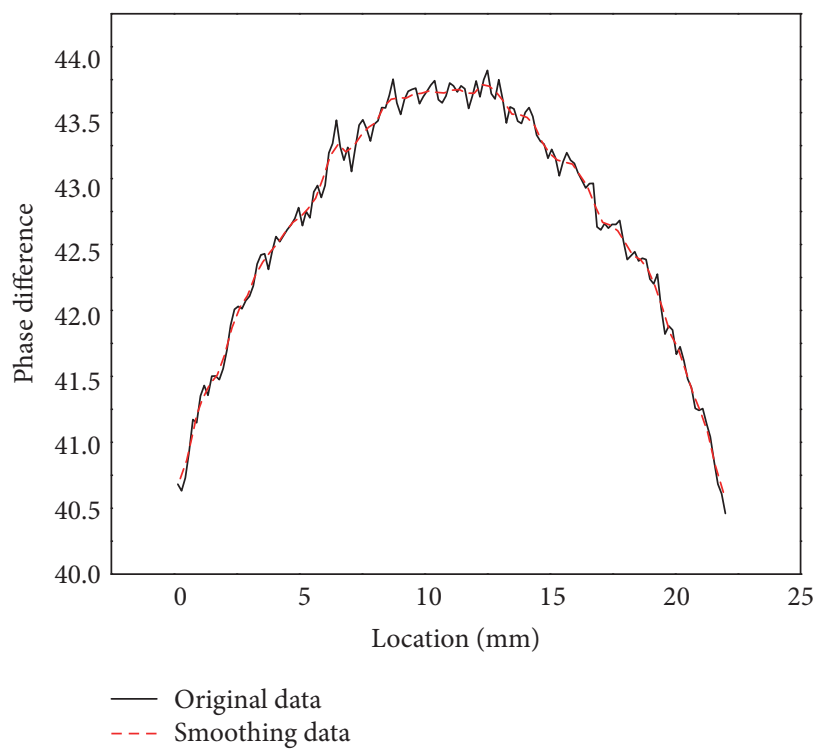

Figure 7: Phase difference of different layers of monolayer directional PMMA.
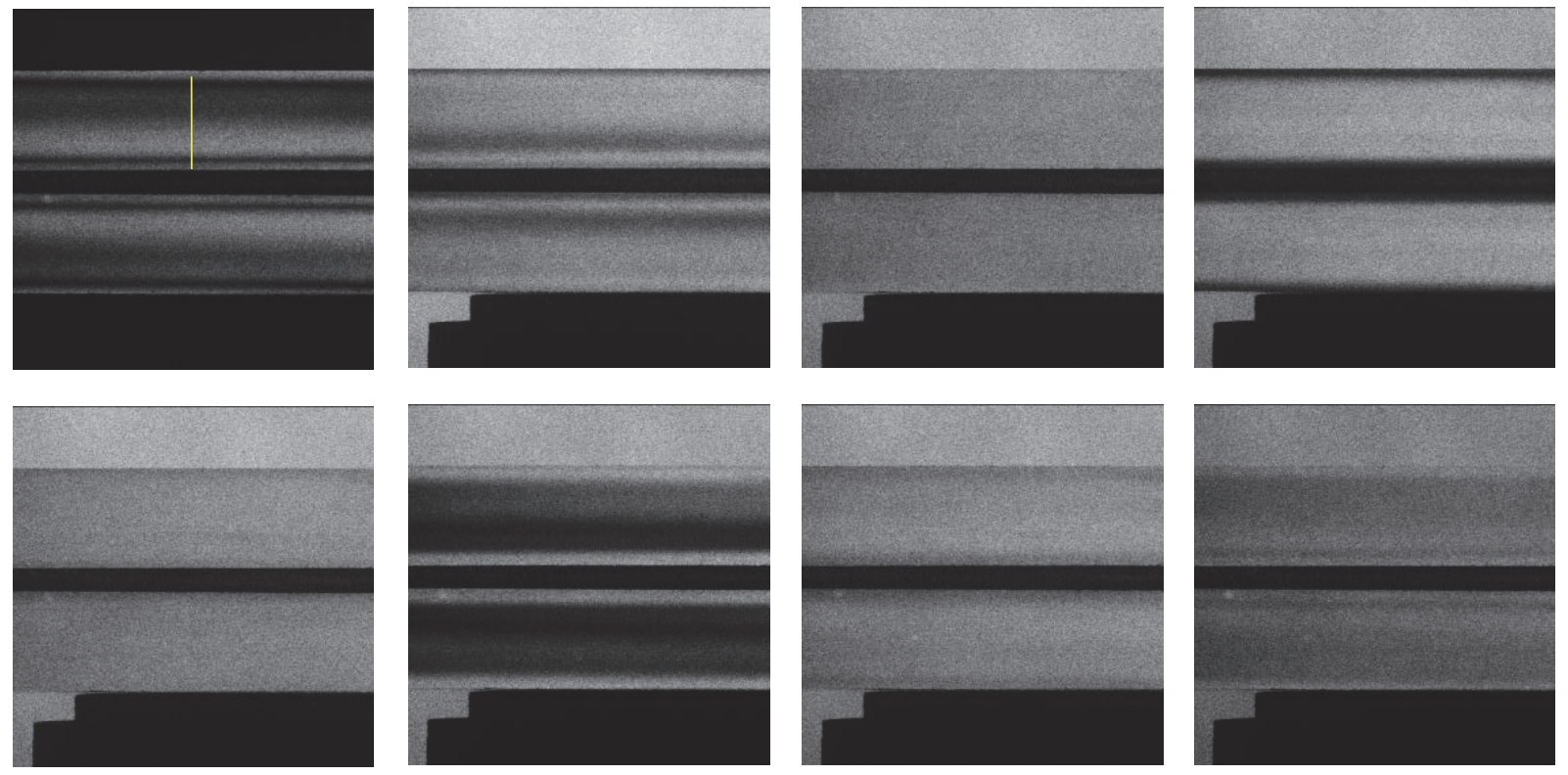

FIGURE 8: Phase-shifting photoelastic images of laminated directional PMMA.

the thickness was $22 \mathrm{~mm}$, and the radius of the notch was $1 \mathrm{~mm}$. Moreover, the thicknesses of upper and lower layers were both $10 \mathrm{~mm}$, while the thickness of the polyurethane interlayer was $2 \mathrm{~mm}$. Figure 8 shows phase-shifting photoelastic images. The digital photoelasticity with the eight-step phase-shifting technique was used to assess the uniformity of the stress in the sample. Result is shown in Figure 9. By analyzing the calculated data, it was seen that since the directional PMMA sheet was reduced to $10 \mathrm{~mm}$ thickness, it was stretched more uniformly than that of the monolayer sheet at a thickness of $22 \mathrm{~mm}$ shown in Figure 7.

The fatigue testing was performed on laminated directional PMMA. Because different layers of laminated directional PMMA were stretched more uniformly than that of monolayer directional PMMA, the interlaminar strength of laminated directional PMMA was enhanced. Moreover, compared with directional PMMA, the elastic modulus of the polyurethane material used as the adhesive interlayer was small, so that the deformation mainly occurred in the polyurethane layer in the middle. Thus the interlaminar strength of laminated directional PMMA was improved.

By analyzing these failure modes of monolayer and laminated directional PMMA specimens, it is found that the monolayer directional PMMA cracked along the tilting direction with many bifurcations shown in Figure 10(a). These bifurcations indicate that the crack grew randomly. This phenomenon may be caused by the weak interlaminar strength of the sample. When the crack grows to a certain 


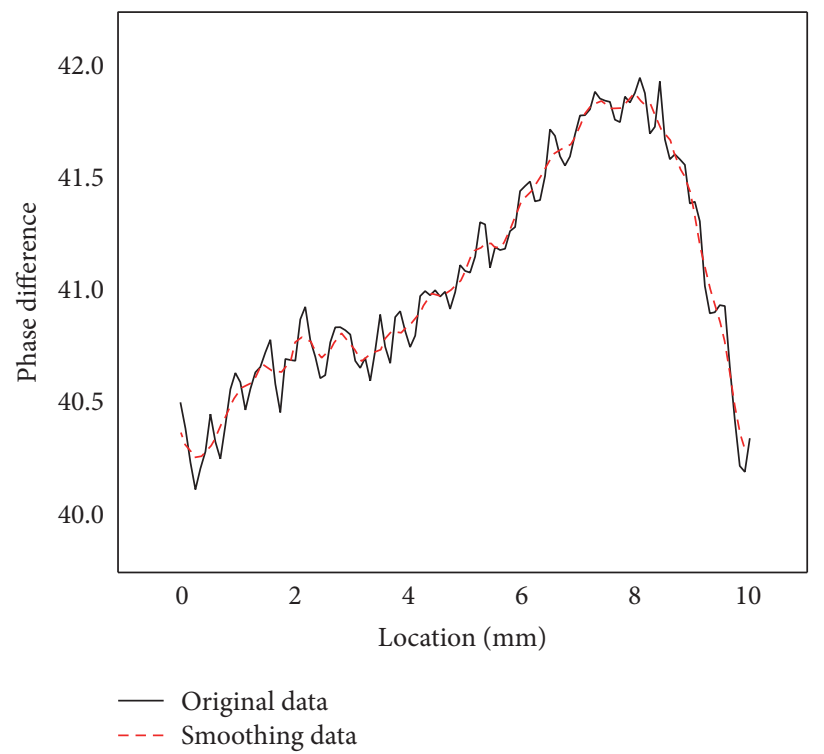

Figure 9: Phase difference of different layers of laminated directional PMMA.

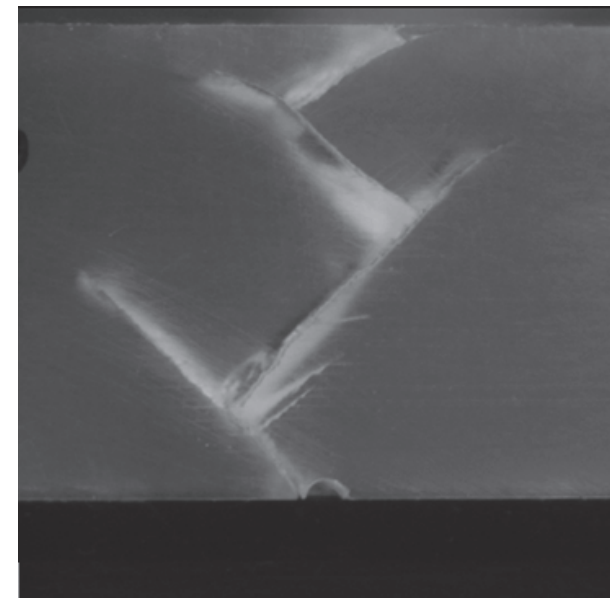

(a)

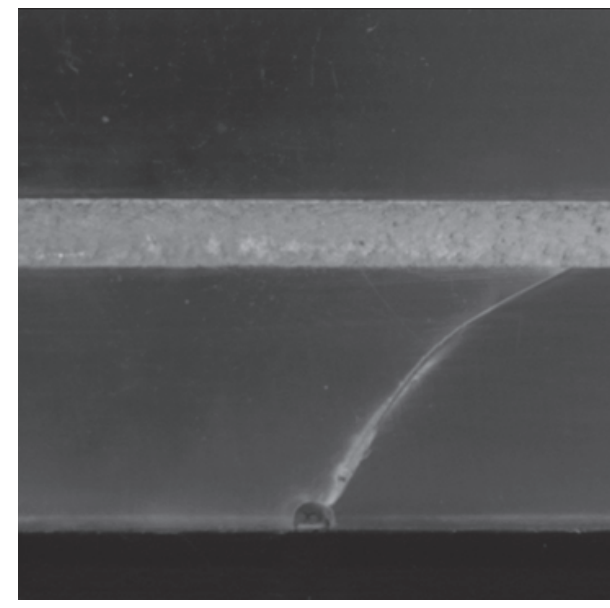

(c)

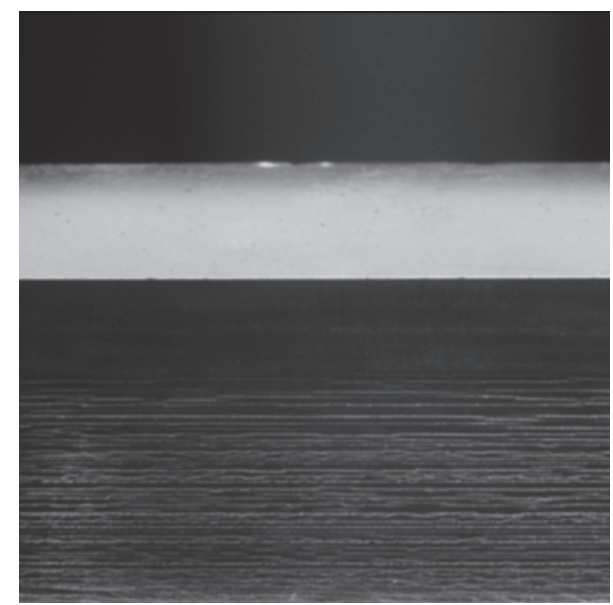

(b)

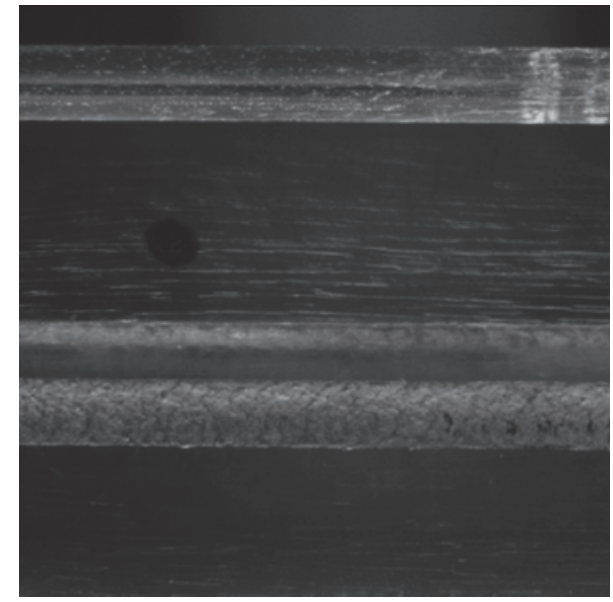

(d)

FIgURE 10: Damage images of monolayer and laminated directional PMMA. (a) Crack propagation of monolayer directional PMMA; (b) surface microcracks of monolayer directional PMMA; (c) crack propagation of laminated directional PMMA; (d) surface microcracks of laminated directional PMMA. 
position, it can grow along two directions. Generally, the crack propagates along the direction with less strength, which causes the bifurcations. Before the crack grows to this extent, with the increase in the number of fatigue cycle applied, microcracks appear in the interlayer shown in Figure 10(b), which causes the cracks to grow towards the interlamination. However, although the cracks in the laminated directional PMMA sample grew along the tilting direction, there was no crack bifurcation shown in Figure 10(c). The reason is that the crack quickly grows to the weak adhesive area. The laminated directional PMMA still retains the certain carrying capacity. Moreover, the density of interlayer microcracks in laminated directional PMMA is lower than that in the monolayer directional PMMA sample shown in Figures 10(b) and $10(d)$. In addition, the fatigue life of laminated directional PMMA is higher than that of monolayer directional PMMA. All results prove that the lamination can improve the fatigue fracture properties of directional PMMA.

\section{Conclusion}

The main purpose of the present work is to investigate fatigue fracture mechanical properties of aeronautical PMMA which has been treated by the directional tensile technology. This research tracked the growth of fatigue cracks in directional PMMA by a stroboscopic method. In this way, gray distribution images of the surface of the specimen at the same moment in different cycles can be acquired. In the area with the concentrated stress, fatigue cracks tended to grow along the direction of tilt rather than in the vertical direction.

The internal stress in directional PMMA toughened by the stretching process reflects the uniformity of the tension for directional PMMA. This research investigated the phase difference in directional PMMA using the digital photoelasticity with the eight-step phase-shifting technique. Because the internal stress is related to the phase difference, the phase difference was used to characterize the uniformity of the tension. Results indicate that different layers of the directional PMMA sheet were stretched to different extents. Under such circumstances, mechanical properties of each layer are inconsistent, which may cause the interlaminar cracking. As a result, the interlaminar strength is decreased. This phenomenon can explain the fatigue cracks growth along the tilting direction rather than in the vertical direction. In addition, directional PMMA was stretched at a temperature below its glass transition temperature, so molecular chains along the stretching direction became ordered and oriented. Mechanical properties of directional PMMA were improved. However, since molecular chains were not stretched along the thickness direction of the sheet, the strength was lower in that direction, which caused the growth of the fatigue crack along the tilting direction.

This work also studied the fatigue fracture properties of laminated directional PMMA and compared these properties with those of monolayer directional PMMA. Results show that the lamination technology can strengthen the interlayer of directional PMMA and enhance its fatigue fracture properties.

\section{Competing Interests}

The authors declare that there are no competing interests regarding the publication of this article and regarding the funding that they have received.

\section{Acknowledgments}

The authors acknowledge the financial support of the National Natural Science Foundation of China (Grants nos. 11402166, 51301057, 51208336, and 51408400) and the General Projects in Tianjin Research Program of Application Foundation and Advanced Technology (Grants nos. 13JCYBJC39100 and 14JCYBJC21900).

\section{References}

[1] J. A. Sauer, J. Marin, and C. C. Hsiao, "Creep and damping properties of polystyrene," Journal of Applied Physics, vol. 20, no. 6, pp. 507-517, 1949.

[2] C. C. Hsiao and J. A. Sauer, "On crazing of linear high polymers," Journal of Applied Physics, vol. 21, no. 11, pp. 1071-1083, 1950.

[3] B. Mukherjee and D. J. Burns, "Fatigue-crack growth in polymethylmethacrylate," Experimental Mechanics, vol. 11, no. 10, pp. 433-439, 1971.

[4] W. M. Cheng, G. A. Miller, J. A. Manson, R. W. Hertzberg, and L. H. Sperling, "Mechanical behaviour of poly (methyl methacrylate). Part 2 the temperature and frequency effects on the fatigue crack propagation behaviour," Journal of Materials Science, vol. 25, no. 4, pp. 1924-1930, 1990.

[5] H.-S. Kim and Y.-W. Mai, "Effect of temperature on fatigue crack growth in unplasticized polyvinyl chloride," Journal of Materials Science, vol. 28, no. 20, pp. 5479-5485, 1993.

[6] H. S. Kim and X. M. Wang, "Temperature and frequency effects on fatigue crack growth of uPVC," Journal of Materials Science, vol. 29, no. 12, pp. 3209-3214, 1994.

[7] F. Ramsteiner and T. Armbrust, "Fatigue crack growth in polymers," Polymer Testing, vol. 20, no. 3, pp. 321-327, 2001.

[8] X. F. Yao, G. C. Jin, K. Arakawa, and K. Takahashi, "Experimental studies on dynamic fracture behavior of thin plates with parallel single edge cracks," Polymer Testing, vol. 21, no. 8, pp. 933-940, 2002.

[9] X. F. Yao, W. Xu, M. Q. Xu, K. Arakawa, T. Mada, and K. Takahashi, "Experimental study of dynamic fracture behavior of PMMA with overlapping offset-parallel cracks," Polymer Testing, vol. 22, no. 6, pp. 663-670, 2003.

[10] S. Sahraoui, A. El Mahi, and B. Castagnède, "Measurement of the dynamic fracture toughness with notched PMMA specimen under impact loading," Polymer Testing, vol. 28, no. 7, pp. 780783, 2009.

[11] W. Liu, Z. Z. Gao, and Z. F. Yue, "Steady ratcheting strains accumulation in varying temperature fatigue tests of PMMA," Materials Science and Engineering A, vol. 492, no. 1-2, pp. 102109, 2008.

[12] W. Shang, X. Yang, and L. Zhang, "Study of anisotropic mechanical properties for aeronautical PMMA," Latin American Journal of Solids and Structures, vol. 11, no. 10, pp. 1777-1790, 2014.

[13] R. W. T. Preater, "Pulsed laser ESPI applied to particular rotating component problems," in Proceedings of the Optics in Engineering Measurement, vol. 599 of Proceedings of SPIE, pp. 174-177, Cannes, France, November 1985. 
[14] J. P. Sikora, "Deflection of rotating marine propellers using projected-grating moiré techniques," Experimental Mechanics, vol. 21, no. 12, pp. 456-460, 1981.

[15] M. R. Hart, R. A. Conant, K. Y. Lau, and R. S. Muller, "Stroboscopic interferometer system for dynamic MEMS characterization," Journal of Microelectromechanical Systems, vol. 9, no. 4, pp. 409-418, 2000.

[16] C. Rembe, B. Tibken, and E. P. Hofer, "Analysis of the dynamics in microactuators using high-speed cine photomicrography," Journal of Microelectromechanical Systems, vol. 10, no. 1, pp. 137145, 2001.

[17] X.-C. Zhang, H.-W. Ji, and Z.-X. Qiao, "Residual stress in selfhealing microcapsule-loaded epoxy," Materials Letters, vol. 137, pp. 9-12, 2014.

[18] W. Shang, X. Ji, and X. Yang, "Study on several problems of automatic full-field isoclinic parameter measurement by digital phase shifting photoelasticity," Optik, vol. 126, no. 19, pp. 19811985, 2015.

[19] P. Pinit and E. Umezaki, "Digitally whole-field analysis of isoclinic parameter in photoelasticity by four-step color phaseshifting technique," Optics and Lasers in Engineering, vol. 45, no. 7, pp. 795-807, 2007. 

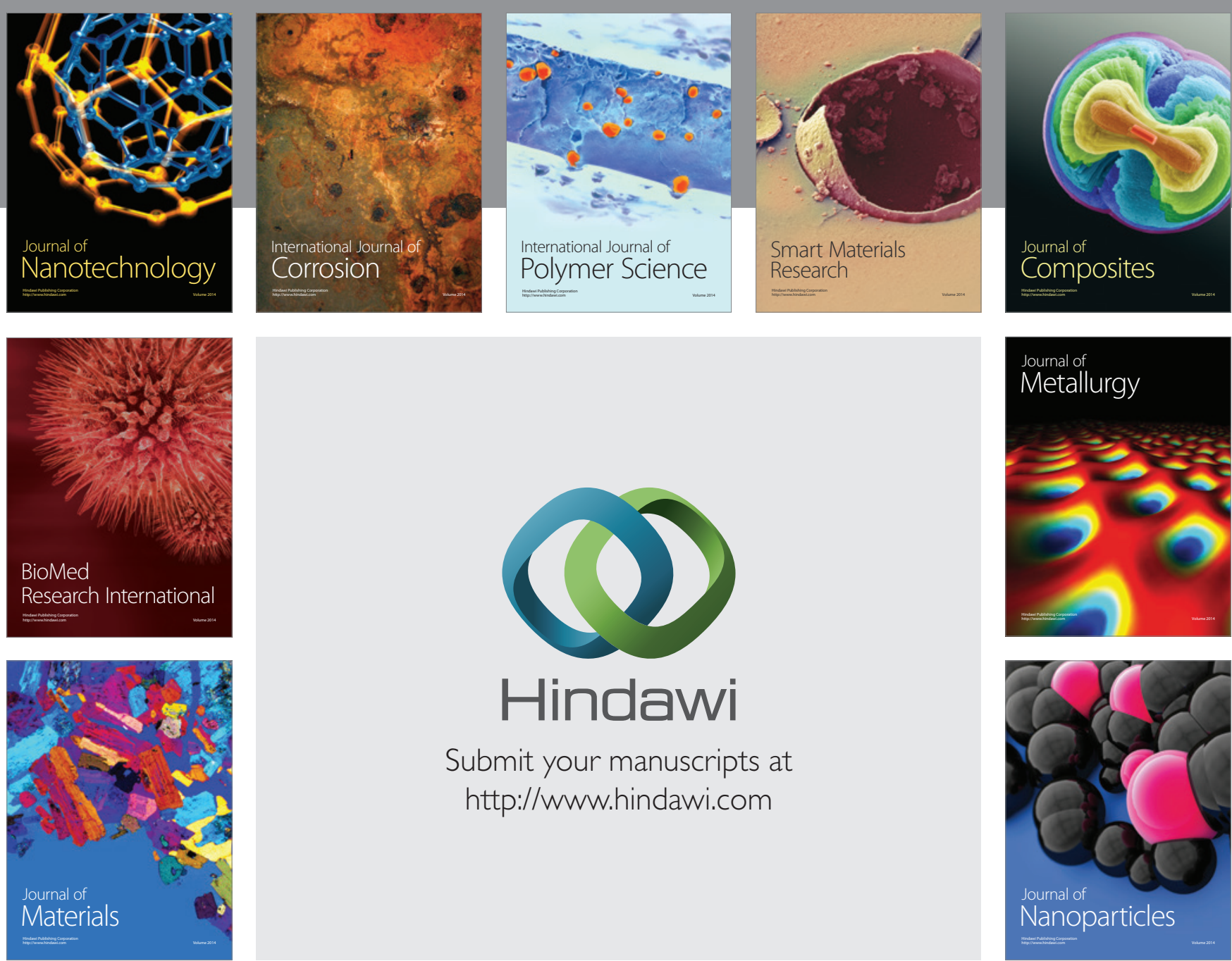

\section{Hindawi}

Submit your manuscripts at

http://www.hindawi.com

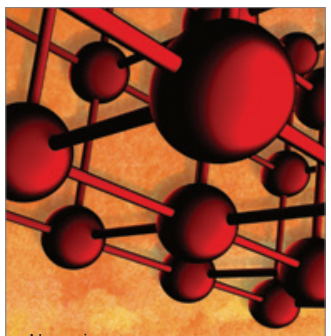

Materials Science and Engineering
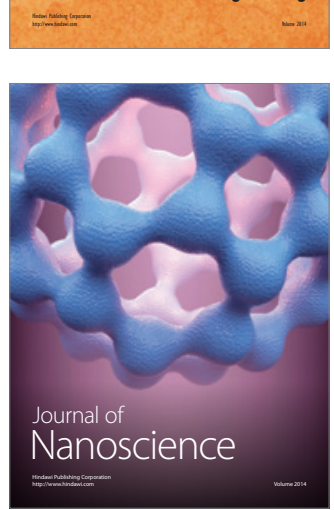
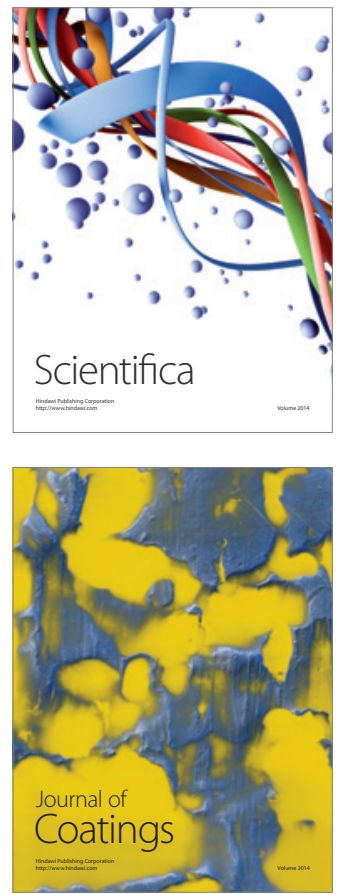
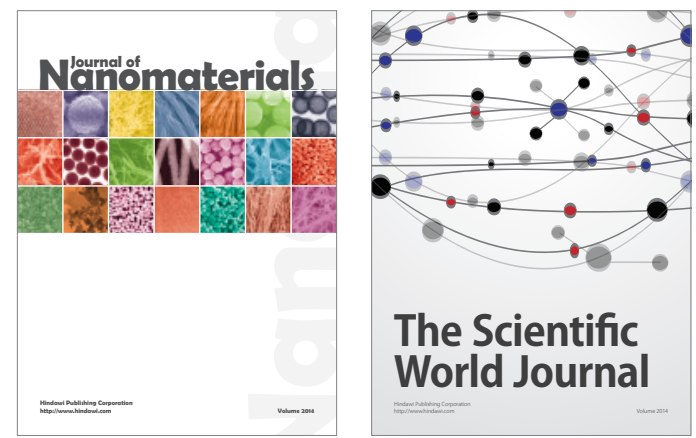

The Scientific World Journal
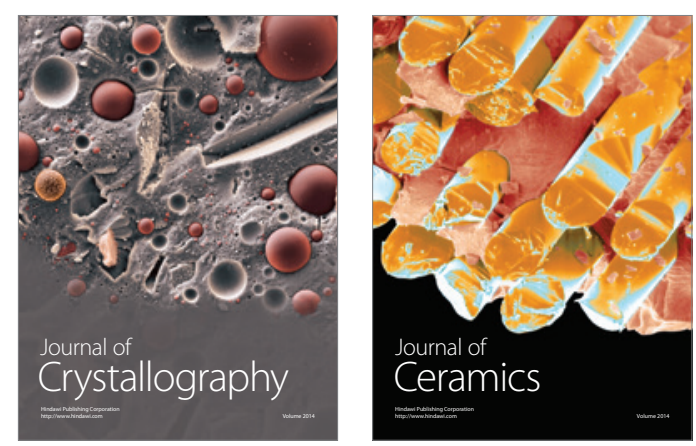
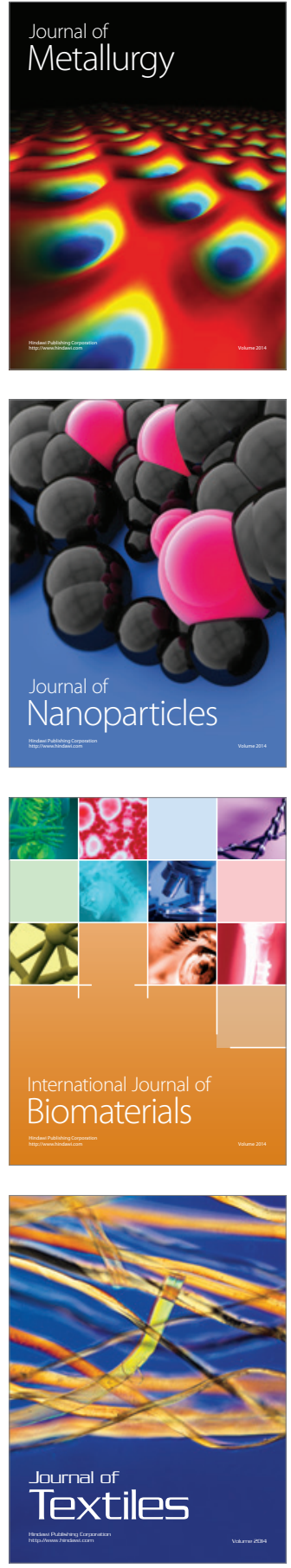